\title{
Caracterización del dolor osteomuscular y apoyo social percibido en adultos mayores de la ciudad de Bucaramanga, Colombia²
}

\section{Ara Mercedes Cerquera Córdoba \\ Doctora en Ciencias Psicológicas Universidad Pontificia Bolivariana Correo electrónico: ara.cerquera@upb.edu.co}

\section{Ana Fernanda Uribe Rodríguez \\ Doctora en Psicología Clínica y de la Salud Universidad Pontificia Bolivariana \\ Correo electrónico: anafernanda.uribe@upb.edu.co}

\section{Yeferson Jahir Matajira Camacho \\ Especialista en Psicología Clínica Universidad Pontificia Bolivariana \\ Correo electrónico: yeferson.matajira@upb.edu.co}

\section{María Alejandra Salas Catalán}

Psicóloga

Universidad Pontificia Bolivariana

Correo electrónico: maria.salas.c@hotmail.com
Recibido: 04/04/2018

Evaluado: $10 / 04 / 2018$

Aceptado: 05/09/2018

\section{Resumen}

Objetivo: Identificar las diferentes características del dolor osteomuscular y la percepción del apoyo social en una muestra de adultos mayores de la ciudad de Bucaramanga. Método: Se trabajó un enfoque metodológico cuantitativo, descriptivo, con una muestra por conveniencia de 120 adultos mayores del departamento de Santander, Bucaramanga, Colombia. Resultados: Se encontró que la muestra estaba compuesta por mujeres, en un $73.3 \%$; la mayoría entre los 65 a 75 años. En cuanto a las características de la afección, el 78\% desconoce su diagnóstico. Predomina una descripción del dolor como punzante, cruel y molesto. Respecto al apoyo social se puede observar que la muestra presenta altos niveles en las categorías apoyo afectivo $78 \%$, apoyo instrumental $76 \%$, interacción social 73\% y apoyo informacional 72\%. Conclusión: Se evidencian diferentes descriptores paras las experiencias de dolor, así como un alto apoyo social percibido. Se sugiere realizar análisis correlacionales para identificar si el apoyo social media en la experiencia del dolor como lo refieren diversas investigaciones.

\section{Palabras clave}

Dolor crónico, Adulto mayor, Apoyo social, Envejecimiento.

2 Para citar este artículo: Cerquera, A., Uribe, A., Matajira, Y. \& Salas, A. (2019). Caracterización del dolor osteomuscular y apoyo social percibido en adultos mayores de la ciudad de Bucaramanga, Colombia. Informes Psicológicos, 19(2), pp. 25-41 http://dx.doi.org/10.18566/infpsic.v19n2a02 


\section{Characterization of musculoskeletal pain and perceived social support in older adults in the city of Bucaramanga, Colombia}

Abstract

Objective: To identify the different characteristics of musculoskeletal pain and the perception of social support in a sample of older adults in the city of Bucaramanga. Method: A quantitative, descriptive methodological approach was used, with a convenience sample of 120 older adults from the department of Santander, Bucaramanga, Colombia. Results: It was found that the sample was composed of women, in $73.3 \%$, most of whom were between 65 and 75 years old. Regarding the characteristics of the condition, $78 \%$ do not know their diagnosis. A description of pain as being sharp, cruel and annoying predominates. Regarding social support, it can be observed that the sample presents high levels in the following categories: affective support (78\%), instrumental support (76\%), social interaction (73\%) and informational support (72\%). Conclusion: Different descriptors are evidenced for pain experiences, as well as a high perceived social support. It is suggested to perform correlational analyses to identify if the social support mediates pain experience as referred to in various investigations.

Keywords chronic pain, older adult, social support, aging.

\section{Caracterização da dor osteomuscular e 0 apoio social percebido em idosos da cidade de Bucaramanga, Colômbia}

Resumo

Objetivo: identificar as diferentes características da dor osteomuscular e a percepção do apoio social em uma amostra de idosos da cidade de Bucaramanga. Metodologia: no trabalho usou-se um enfoque metodológico quantitativo, descritivo, com uma amostra de conveniência de 120 idosos do departamento de Santander, Bucaramanga, Colômbia. Resultados: verificou-se que a amostra esteve composta por mulheres em um $73.3 \%$, a maioria entre 65 e 75 anos. Em relação às características da condição, $78 \%$ desconhecem seu próprio diagnostico, predomina uma descrição da dor como aguda, cruel e irritante. Em relação ao apoio social pode-se observar que a amostra apresenta altos níveis nas categorias de apoio afetivo com um $78 \%$, apoio instrumental com um $76 \%$, interação social com um $73 \%$ e suporte informacional em um $72 \%$. Conclusão: evidenciam-se diversos descritores para as experiências da dor, bem como um alto apoio social percebido, é sugerido continuar com a realização de análises correlacionais para identificar se 0 suporte social tem mediação na experiência da dor como é referido em várias pesquisas.

Palavras chave dor crônica, idoso, apoio social, envelhecimento. 


\section{ntroducción}

Uno de los síntomas más frecuentes en las consultas médicas en la población mayor es el dolor, el cual se encuentra relacionado directamente con los procesos del envejecimiento primario y secundario (CEPAL, 2006; Corujo \& Pérez, 2007; Gibson, 2007; Helme \& Gibson, 2001). Lo anterior lo ratifican estudios internacionales que afirman que la prevalencia del dolor en adultos de 65 años es de un 25\% y $88 \%$, indicando que los adultos mayores, son más propensos a manifestar dolor en comparación con otros grupos poblacionales (Brennan, Carr \& Cousins, 2007; Català et al., 2002; Cowan, Fitzpatrick \& Roberts, 2003; Gloth, 2000; Herr \& Garand, 2001; Helme \& Gibson, 2001).

Como concepto, el dolor es definido por la Asociación Internacional para el estudio del dolor (IASP, 1994) como una experiencia sensitiva y emocional desagradable relacionada a un daño tisular real o posible, el cual tiene una manifestación a nivel fisiológico, autonómico y conductual (Bader et al., 2010; Plata \& Cerquera, 2016; Terman \& Bonica, 2001); al mismo tiempo, la percepción de éste suele afectarse por diversos factores, tales como las bases culturales y la experiencia emocional de afrontamiento y enfrentamiento vivida ante el dolor; por esta razón, la tolerancia y el umbral del dolor, son distintas en cada individuo, incluso en la misma persona en diferentes circunstancias (Maestre, 2001; Sánchez, 2003; Trujillo, 2008).

Se considera que en Estados Unidos existe entre un 35 a $48 \%$ de adultos mayores que experimentan dolor a diario de tipo crónico (Krueger \& Stone, 2008; Maxwell et al., 2008), el cual se caracteriza por tener una duración de más de tres meses, su permanencia puede tener diferentes factores del causante original del dolor, biológicamente es apreciado como no útil, es continuo y desproporcionado y se considera poco propenso a la curación (IASP,1994; Martínez-Salio, 2007; Soto, 2011).

A partir de ello, se ha encontrado en diversas investigaciones que, en gran medida, los adultos mayores de setenta y cinco años de Estados Unidos refirieron haber sentido alguna clase de dolor y cerca de dos terceras partes de este mismo grupo de adultos mayores institucionalizados, padecen dolor crónico (American Geriatrics Society, 2002; Clark \& Cox, 2002).

Así mismo, estudios en adultos mayores con edades superiores a 65 años, evidenciaron que la prevalencia del dolor crónico en las mujeres era mayor que en hombres (Álvarez-Namegyei, Nuño-Gutiérrez \& Alcocer-Sánchez, 2005; Covarrubias-Gómez et al., 2008).

En otro estudio realizado en Colombia en la ciudad de Manizales, se demostró que la prevalencia de dolor en un grupo de adulto mayores, fue del 50\% y de dolor crónico del 31\% (Díaz, Marulanda \& Sáenz, 2009). Así mismo, la Asociación Colombiana para el Estudio del Dolor (ACED) en la VIII Encuesta Nacional de dolor realizada en el 2014, indicaba que la prevalencia de esta afección en los últimos 6 meses de la muestra seleccionada, fue del $76 \%$ y el $47 \%$ de los participantes. En la encuesta, se considera el dolor osteomuscular 
como aquel que presentan compromisos a nivel de huesos, tendones y articulaciones. En relación con el mismo, se encontró que el $64.5 \%$ de los orígenes de dolor están asociados con el sistema osteomuscular.

Esta afección de igual manera tiende a presentarse con mayor frecuencia en las mujeres e incrementa con la edad. De la misma forma, se ha encontrado una alta comorbilidad en personas que se encuentran casadas o viudas y con nivel socioeconómico bajo. Dentro de las enfermedades más frecuentes se encuentran la artrosis y las lumbalgias crónicas (López \& López, 2012). Lo anterior guarda relación con los estudios de Noceda, Moret y Lauzirika (2006a) quienes han evidenciado en sus investigaciones que el tipo de dolor crónico osteomuscular más relevante es la artrosis, la cual se presentaba en un $72.6 \%$ en los mayores de 65 años. Además, se observó cómo la mayor parte de los pacientes presentaban dolor crónico con más de 24 meses de duración, encontrándose una marcada afectación sobre su calidad de vida (Noceda, Moret \& Lauzirika, 2006b)

Por otra parte, en un estudio desarrollado en mujeres trabajadoras mexicanas, se demostró que tanto el apoyo de la familia como del trabajo, desempeñan un papel fundamental en la forma en que el dolor crónico osteomuscular es afrontado, lo cual hace visible el valor tan importante que representan las redes de apoyo primarias del doliente (Ordóñez-Hernández, Contreras-Estrada \& Soltero-Avelar, 2015).

Como concepto, el apoyo social es definido como toda acción de tipo emocional, instrumental e informacional, cognitiva y material, realizada por diferentes actores, con el fin de cooperar en el bienestar de otra persona (Guzmán, Huenchuan \& Montes de Oca, 2003; Matud, Carballeira, López, Marrero \& Ibáñez, 2002). Dichas acciones son establecidas a partir de una red social, previamente constituida por un grupo de personas; diferentes estudios han demostrado que la prevalencia y la funcionalidad de estas redes, están directamente relacionadas con mejores experiencias de vida y con mejores condiciones de salud y bienestar tanto en la población general, como en los adultos mayores (Curcio-Borrero, 2008; Gallegos-Carrillo et al., 2009; Peláez \& Rodríguez-Wong, 2004; Pelcastre-Villafuerte, Treviño-Siller, González-Vázquez \& Márquez-Serrano, 2011).

En un estudio realizado en Chile, donde se analizó las relación que presentaba el bienestar psicológico, el apoyo social y la percepción del estado de salud entre adultos mayores de 60 a 87 años, se encontró que los participantes casados o con una pareja estable, presentaban altos niveles de bienestar psicológico y percepción de apoyo social que aquellos que estaban solteros o sin pareja; así mismo, el bienestar psicológico está más relacionado con el apoyo social percibido que con las percepciones de la salud de los participantes (Vivaldi \& Barra, 2012). En una investigación donde se exploró la relación entre el apoyo social y la sintomatología depresiva en personas mayores chilenas, se demostró que las estructuras familiares nucleares, funcionales, son las principales fuentes de apoyo para mitigar los síntomas depresivos, los cuales, según Bistre (2007) y McWilliams, Brian \& Murray 
(2003), en el 50\% de las personas con experiencia de dolor se presentan, junto con otras sintomatologías como ansiedad, alteraciones en el ciclo del sueño, dependencia funcional, disminución de los procesos de socialización y actividades de ocio generando una disminución en la calidad de vida (Herr \& Garand, 2001; Robinson, 2007).

De la misma manera, cuando los vínculos entre ellos se rompen, se puede experimentar sentimientos de soledad que generan una percepción del apoyo social negativo (Gallardo-Peralta, Sánchez-Moreno, Arias-Astray \& López-deRoda, 2015; Jaswal \& Sing, 2014); por ende, se ven alteradas las relaciones intrafamiliares, amistosas y de pareja, así como también las actividades cotidianas (Amaya-Ropero \& Carrillo-González, 2015; De Andrés, Acuña \& Olivares, 2014).

Ferreira y Sherman (2007) quienes exploraron la relación entre el optimismo, el dolor y el apoyo social y su relación con el bienestar general de adultos mayores con padecimiento de osteoartritis, encontraron que el apoyo social fue un mediador entre el dolor y los síntomas depresivos de los participantes. De igual manera, se evidenció que, en aquellos pacientes con mayor dolor y que perciben menos apoyo social, había un incremento en los síntomas depresivos. Por otro lado, en un estudio sobre el estado de salud percibido, la satisfacción vital y el efecto moderador del apoyo social de 406 adultos mayores españoles se encontró que el apoyo social tuvo un efecto moderador en el impacto que la salud percibida tiene sobre la satisfacción vital de los participantes. En particular, aquellos participantes que indicaron tener una condición de salud muy mala o mala, percibieron un adecuado apoyo social (Dumitrache, Rubio \& Rubio-Herrera, 2016).

Por otra parte, en un estudio longitudinal en el cual se investigó la relación entre el estrés psicológico en pacientes recientemente diagnosticados con artritis reumatoide se encontró que mientras más alto es el apoyo social y mientras menor es el dolor, menor sería el estrés psicológico de los pacientes (Benka et al., 2012). Lo anterior guarda relación con el estudio realizado por Ma et al. (2015) en el cual se demostró correlaciones positivas entre el apoyo social y calidad de vida en adultos mayores con osteoporosis. Mientras que para autores como Franks, Cronan y Oliver (2004) quienes analizaron los efectos tanto de la cantidad como de la calidad del apoyo social en el bienestar psicológico y físico de un grupo de mujeres pacientes de fibromialgia, encontraron que la calidad del apoyo social resulta más importante que la cantidad del mismo. Por lo anterior, el objetivo de la presente investigación es identificar las diferentes características del dolor osteomuscular y la percepción del apoyo social en una muestra de adultos mayores

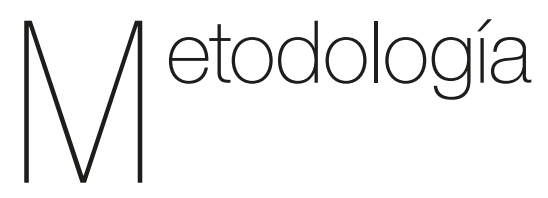

Se trabajó con un enfoque metodológico cuantitativo, descriptivo, con una muestra a conveniencia de 120 adultos mayores de la ciudad de Bucaramanga, Colombia. 


\section{Criterios de inclusión}

- $\quad$ Edad mayor o igual a 60

- Capacidad para responder a los cuestionarios

- Adultos mayores que refieran padecer dolor osteomuscular.

\section{Aspectos éticos}

Para la presente investigación se guardó la confidencialidad de los participantes mediante la firma del documento del consentimiento informado en el cual, también se daba claridad sobre los objetivos y características de la investigación como lo determina la Ley 1090 de 2006, numeral 9 del artículo 2.

\section{Instrumentos}

Entrevista semiestructurada: Elaborada por los investigadores para indagar sobre variables sociodemográficas presentes en las características de la muestra seleccionada: se preguntó sobre el género, edad, estado civil, situación laboral, nivel de ingreso, nivel escolar y diagnóstico. La última variable hace referencia al conocimiento diagnóstico que tenía el adulto mayor sobre las causas de sus dolencias. Se analizó si la muestra tenía algún conocimiento a partir de su historial médico, sobre las causas de su afección.

\section{Cuestionario de Dolor de McGill}

(Bejarano, Osorio, Rodríguez \& Berrío, 1985). Está compuesto por 62 adjetivos distribuidos en 20 grupos, que, a su vez, se clasifican en dimensiones que contienen de 2 a 4 opciones de respuesta.

Tabla 1.

Dimensiones del McGill

\begin{tabular}{|c|c|c|c|}
\hline a. & Sensorial & (S) & $(1-10)$ \\
\hline b. & Afectivo & (A) & $(11-15)$ \\
\hline c. & Evaluativo & (E) & (16) \\
\hline d. & Miscelánea sensorial & (MS) & $(17-19)$ \\
\hline e. & Miscelánea afectiva & (MAE) & (20) \\
\hline f. & Miscelánea total & (MT) & $(17-20)$ \\
\hline \multicolumn{4}{|c|}{ Además del indice total del dolor: } \\
\hline \multicolumn{2}{|c|}{ Indice total } & (IT) & $(1-20)$ \\
\hline
\end{tabular}

En cuanto a las opciones de respuesta, cada una tiene asignada un rango de acuerdo a las palabras escogidas. De esta manera, se obtiene el "Índice de Valoración del Dolor" la cual refleja la forma como el sujeto califica su experiencia dolorosa desde factores emocionales y sensoriales. La puntuación de cada dimensión se obtiene teniendo en cuenta las palabras que el sujeto selecciona como descriptores de su dolor en cada categoría, de acuerdo al orden que tienen el grupo al que pertenecen. En la Tabla 2 se informa la estadística descriptiva de las dimensiones del MPQ en personas con dolor crónico por los autores de la validación colombiana. 
Tabla 2.

Estadística descriptiva de las puntuaciones en las dimensiones del MPQ de la muestra de personas con dolor crónico

\begin{tabular}{llll}
\hline Crónicos & & & \\
\hline Sensorial (1-10) & $\mathrm{n}=150$ & $\overline{\mathrm{x}} 17,17$ & $\mathrm{DE}=12.12$ \\
Afectiva (11-15) & $\mathrm{n}=150$ & $\overline{\mathrm{x}} 7,27$ & $\mathrm{DE}=2.20$ \\
Evolutiva (16) & $\mathrm{n}=150$ & $\overline{\mathrm{x}} 2,16$ & $\mathrm{DE}=1.36$ \\
Miscelánea (17-20) & $\mathrm{n}=150$ & $\overline{\mathrm{x}} 6,19$ & $\mathrm{DE}=3.05$ \\
Puntaje total (1-20) & $\mathrm{n}=150$ & $\overline{\mathrm{x}} 31,83$ & $\mathrm{DE}=6.90$ \\
\hline
\end{tabular}

Fuente: Bejarano et al. (1985)

\section{Cuestionario MOS de apoyo social percibido.}

Este cuestionario fue validado en Colombia por Londoño et al. (2012). Tiene por objetivo evaluar el apoyo social percibido. Contiene 20 preguntascon opción de respuesta tipo Likert, cuyas opciones de respuesta son: Nunca (1), Pocas veces (2), Algunas veces (3), La mayoría de las veces (4), Siempre (5); de manera que, a mayor puntuación, mayor apoyo social percibido.

Los ítems evalúan cuatro tipos de apoyo: apoyo emocional o informacional, que se refiere a la comprensión empática y la guía u oferta de consejos y orientación (preguntas 3, 4, 8, 9, 13, 16, 17, 19; punto de corte: 24 puntos); apoyo instrumental, es decir, la provisión de ayuda material que pueda precisar la persona (preguntas: 2, 5, 12, 15; punto de corte:12 puntos); interacción social positiva, referida a la disponibilidad de personas con las cuales salir, divertirse o distraerse (preguntas 7, 11, 14, 18; unto de corte: 12 puntos); y apoyo afectivo, la expresión de amor y afecto (preguntas 6, 10, 20; punto de corte: 9 puntos). La confiabilidad de la escala global, medida a través del alfa de Cronbach fue de .94, y la de sus componentes osciló entre .92 y .73.

\section{Procedimiento}

Inicialmente se contactó a los participantes a conveniencia. Como estrategia, se realizó la divulgación del proyecto por medio de los líderes de los diferentes grupos de adulto mayor, quienes trabajaban con la población diana en los diversos parques del área metropolitana. A partir de la información recopilada, se creó una base de datos con el fin de llevar un registro riguroso y organizado de los datos, dando inicio de tal modo al contacto con la muestra mediante medio telefónico. Una vez contactado al participante, se le dio a conocer el objetivo de la investigación y toda la información ética y profesional que representaba; por ende, si el participante señalaba que le interesaba participar de la investigación, se procedía a estipular una fecha para que el investigador y el participante se reunieran y se aplicara la batería de pruebas. Cabe resaltar que, en esta parte del proceso, se indaga al mismo tiempo con el entrevistado si refiere padecer algún tipo de dolor osteomuscular. Si la respuesta es afirmativa se procede con el siguiente paso.

En el momento de encuentro con el participante, se le dio a conocer nuevamente los lineamientos éticos y 
procedimentales de la investigación, así como los objetivos y los instrumentos de evaluación a aplicar, tales como la entrevista semiestructurada, el cuestionario MOS de apoyo social percibido y el Cuestionario de Dolor de McGill.
Aplicadas las pruebas al número de participantes señalados para la investigación, se inició con el proceso de tabulación de datos en formato Excel. Realizada la tabulación, se llevó a cabo el correspondiente análisis de los datos por medio del programa SPSS, versión 22.

\section{Resultados}

Tabla 3.

Datos sociodemográficos

\begin{tabular}{|c|c|c|c|}
\hline Descripción & & $\mathbf{F}$ & $\%$ \\
\hline \multirow{2}{*}{ Sexo } & Masculino & 32 & 26.7 \\
\hline & Femenino & 88 & 73.3 \\
\hline \multirow{4}{*}{ Edad } & 65 a 75 años & 83 & 69.2 \\
\hline & 76 a 84 años & 33 & 2.5 \\
\hline & 85 años hacia adelante & 4 & 3.3 \\
\hline & Casado & 52 & 43.3 \\
\hline \multirow{5}{*}{ Estado civil } & Soltero & 17 & 14.2 \\
\hline & Viudo & 38 & 31.7 \\
\hline & Divorciado & 4 & 3.3 \\
\hline & Unión no formalizada & 4 & 3.3 \\
\hline & Separado & 5 & 4.2 \\
\hline \multirow{6}{*}{ Vínculo laboral } & V. Laboral estable & 6 & 5.0 \\
\hline & V. laborar inestable & 9 & 7.5 \\
\hline & Pensionado jubilado & 27 & 22.5 \\
\hline & Actividad no lucrativa & 2 & 1.7 \\
\hline & Tareas propias del hogar & 39 & 32.5 \\
\hline & Otro & 37 & 30.8 \\
\hline \multirow{8}{*}{ Escolaridad } & No sabe leer ni escribir & 5 & 4.2 \\
\hline & No terminó Básica Primaria & 45 & 37.5 \\
\hline & Básica Primaria terminada & 43 & 35.8 \\
\hline & No terminó Enseñanza Media & 10 & 8.3 \\
\hline & Terminó Enseñanza Media & 8 & 6.7 \\
\hline & Enseñanza Media Superior & 6 & 5.0 \\
\hline & Universitario & 2 & 1.7 \\
\hline & Desconoce & 1 & .8 \\
\hline \multirow{10}{*}{ Diagnóstico } & Artrosis & 15 & 12.5 \\
\hline & Osteoartrosis & 1 & .8 \\
\hline & Osteoporosis & 18 & 15.0 \\
\hline & Fibromialgia & 2 & 1.7 \\
\hline & Artritis & 3 & 2.5 \\
\hline & Desconoce & 78 & 65.0 \\
\hline & Línea discal & 1 & .8 \\
\hline & Contracción muscular & 1 & .8 \\
\hline & Osteo artritis & 1 & .8 \\
\hline & Descalcificación & 1 & .8 \\
\hline
\end{tabular}

Fuente: autoría propia; Número de participantes muestra = 120 . 
Como se puede observar en la Tabla 3, en la muestra predomina del sexo femenino con un $73.3 \%$. La mayoría se encontraba entre los 65 y 75 años con un $69.2 \%$, mayoritariamente de estado civil casado, con un vínculo laboral actual relacionado con las tareas propias del hogar y con un nivel de escolaridad sin finalización de la básica primaria. Respecto a la variable diagnóstico, se evidenció que la mayoría de la muestra desconoce las causas de sus dolencias.

Tabla 4.

Prueba Mcgill. Percepción subjetiva del dolor

\begin{tabular}{|c|c|c|c|}
\hline Dimensión & Percepción & $\mathrm{Fi}$ & $\%$ \\
\hline \multirow{11}{*}{ Sensorial } & Que pulsa & 46 & 38.3 \\
\hline & Brusco & 21 & 17.5 \\
\hline & Que perfora & 42 & 35.0 \\
\hline & Punzante & 60 & 50.0 \\
\hline & Que presiona & 56 & 46.7 \\
\hline & Que estira & 17 & 14.2 \\
\hline & Ardor & 37 & 30.8 \\
\hline & Hormigueo & 36 & 30.0 \\
\hline & Resentido & 43 & 35.8 \\
\hline & Tenso & 43 & 35.8 \\
\hline & Fatigante & 17 & 14.2 \\
\hline \multirow{2}{*}{ Afectiva } & Deprimente & 32 & 26.7 \\
\hline & Enfermante & 33 & 27.5 \\
\hline \multirow{3}{*}{ Evaluativa } & Cruel & 83 & 69.2 \\
\hline & Desesperante & 52 & 43.3 \\
\hline & Molesto & 85 & 70.8 \\
\hline \multirow{3}{*}{ Miscelánea } & Que penetra & 69 & 57.5 \\
\hline & Rígido & 32 & 26.7 \\
\hline & Frio & 19 & 14.2 \\
\hline \multirow{5}{*}{ Intensidad del dolor actualmente } & Leve & 15 & 9.5 \\
\hline & Moderado & 35 & 33.3 \\
\hline & Fuerte & 53 & 44.2 \\
\hline & Intenso & 9 & 7.5 \\
\hline & Insoportable & 8 & 7.5 \\
\hline \multirow{3}{*}{ Actividad diaria } & Mínima & 6 & 5.0 \\
\hline & Normal & 41 & 34.2 \\
\hline & Disminuida & 73 & 60.8 \\
\hline \multirow{3}{*}{ Sueño } & Normal & 60 & 58.3 \\
\hline & Interrumpido & 49 & 35.8 \\
\hline & Sueño & 21 & 5.8 \\
\hline
\end{tabular}

Fuente: autoría propia 
Con relación a la Tabla 4, se identifican diferencias en la percepción del dolor en la muestra, ya que la mayoría de ésta describió su dolor como punzante, cruel y molesto, mientras que, en la dimensión miscelánea, sobresalió la sensación que penetra. Respecto a la intensidad del dolor actual, el $44.2 \%$ refirió ser fuerte y presentar disminución en el desarrollo de las actividades diarias en un $60.8 \%$. En cuanto al sueño, se encontró que el $58.3 \%$ lo calificaron como normal.

Tabla 5.

Cuestionario Mos. Apoyo social percibido

\begin{tabular}{lcll}
\hline Tipos de apoyo & & $\mathrm{Fi}$ & $\%$ \\
\hline \multirow{2}{*}{ Emocional/Informacional } & Adecuado Uso & 82 & 72.0 \\
& Escaso Uso & 38 & 28.0 \\
\hline \multirow{2}{*}{ Instrumental } & Adecuado Uso & 87 & 76.0 \\
& Escaso Uso & 33 & 24.0 \\
\hline \multirow{2}{*}{ Interacción social positiva } & Adecuado Uso & 84 & 73.0 \\
& Escaso Uso & 36 & 27.0 \\
\hline \multirow{2}{*}{ Afectivo } & Adecuado Uso & 89 & 78.0 \\
& Escaso uso & 31 & 22.0 \\
\hline
\end{tabular}

Fuente: autoría propia

En cuanto a los resultados de la Tabla 5, se puede observar que la muestra presenta altos niveles de apoyo social en las categorías apoyo afectivo (78\%), apoyo instrumental (76\%), interacción social (73\%) y apoyo informacional (72\%).

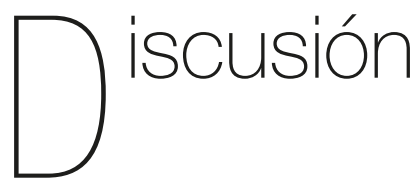

En el presente estudio se encontró que el sexo predominante con altos niveles de dolor crónico, es el femenino con un 73\%. Aunque el muestreo no fue probabilístico, se puede identificar que también en los estudios de López-Mongil y López (2012) las mujeres presentaban en mayor medida presencia de dolor. Por otro lado, el rango de edad más prevalente en el que se encontraba la muestra fue de 65 a 75 años con un 69.2\%; dichos resultados se relacionan con estudios similares en donde indican que existe altos reportes de dolor crónico en mujeres mayores de 65 años; asimismo, se afirma que gran parte de las consultas recibidas por el equipo médico se relacionan con la presencia de dolor osteomuscular o dolor crónico en los adultos mayores, generados principalmente por el proceso de envejecimiento presente en esta etapa del ciclo vital (Álvarez-Namegyei et al., 2005; CEPAL, 2006; Corujo, Pérez, 2007; CovarrubiasGómez et al., 2008; Gibson, 2007; Helme \& Gibson, 2001).

Frente al diagnóstico, el 65\% de los adultos mayores refirieron que no lo conocían ni tampoco la causa de su dolor; lo anterior puede estar relacionado a que 
los servicios de salud brindados al adulto mayor están centrados en evitar o disminuir el deterior funcional, más no en la realización de una valoración médica enfatizada por la presencia de una enfermedad (Villarreal \& Month, 2012). No obstante, según la Asociación Colombiana para el Estudio del Dolor (ACED, 2014) el 64.5\% de los orígenes de dolor en la población adulta mayor están asociados con el sistema osteomuscular. Así mismo, los postulados de autores como Noceda et al. (2006a) mencionan que el tipo de dolor crónico osteomuscular más relevante es la artrosis, la cual se presentaba en un $72.6 \%$ en adultos mayores de 65 años, con una frecuencia alta de manifestación de síntomas. Aunque en la presente investigación se encontró que solo el 12.5\% de la población manifestaba conocer que sus dolencias estaban relacionadas con la artrosis, es necesario tener presente que el hecho de que el 65\% desconozcan el diagnóstico médico por las diferentes condiciones del sistema de salud, no significa que no padezcan objetivamente esta enfermedad u otras.

En cuanto al estado civil y el vínculo laboral, la mayoría de la muestra indicaron estar casados y dedicarse a las labores propias del hogar. Con relación a estos resultados, estudios encontrados han evidenciado que la mayoría de los adultos mayores presentan este estado civil. Respecto a las labores del hogar, guarda una gran relación con lo expuesto por Alpízar (2011) y López-Mongil y López (2012) quienes refieren que el inicio de la vejez tiende a coincidir con la incapacidad para continuar laborando, sobre todo cuando el adulto mayor manifiesta afecciones en su salud. Esta condición en ocasiones lleva a la jubilación anticipada y, por tanto, a tener una mayor disponibilidad de tiempo para realizar actividades propias del hogar o compartir más tiempo con su cónyuge.

Desde los resultados obtenidos en la percepción del dolor crónico, se encontró que en la muestra predomina el dolor punzante, cruel, molesto y con una intensidad fuerte, lo cual guarda relación con los resultados encontrados por Cerquera y Plata (2016) donde se evidenciaron altas puntuaciones en los descriptores incómodo, interno y caliente, así como también en el descriptor de intensidad del dolor fuerte. Frente a lo anterior, se afirma que el dolor crónico contiene algunas características específicas que varían dependiendo del sujeto. Según Maestre (2001), Sánchez (2003) y Trujillo (2008), la percepción del dolor se afecta y se expresa teniendo en cuenta distintos factores como la cultura, la experiencia emocional y el afrontamiento; es por ello que la tolerancia y el umbral del mismo se consideran como una vivencia distinta en cada persona. En otras palabras, factores biológicos, psicológicos y sociales median la experiencia del dolor. Esto guarda relación con diversas investigaciones en las que se ha logrado evidenciar, por ejemplo, mayor sensibilidad al dolor en la adultez y mayor incremento del umbral del dolor en comparación con jóvenes (De Andrés et al., 2014; Torvik, Kaasa, Kirkevold \& Rustoen, 2009); así mismo, representa un factor de vital importancia de análisis, ya que podría evidenciar la presencia de dolor por daño tisular en los adultos mayores.

Finalmente, en cuanto al apoyo social percibido, considerado por Guzmán et al. (2003) como aquellas acciones de tipo emocional, cognitiva y mental realizadas por un individuo, con la intención de 
generar bienestar hacia la otra persona, sin tener en cuenta la situación que está viviendo, en los resultados se evidenció que los adultos mayores refirieron percibir altos niveles de apoyo afectivo informacional, instrumental, de interacción social positiva y de tipo emocional.

Lo anterior guarda relación con los resultados encontrados por Plata y Cerquera (2016) donde se halló que la muestra presentaba altas puntuaciones en las categorías anteriormente expuestas. Esto es un factor de gran importancia, porque el alto apoyo social percibido es un elemento mediador frente a la experiencia del dolor, como lo refieren Ordóñez-Hernández et al. (2015) en sus investigaciones, ya que tanto el apoyo familiar como el laboral desempeñaban un papel fundamental en la forma como el dolor crónico osteomuscular es afrontado.

A partir de este panorama se puede mencionar que, al parecer, como lo refiere Rodrigo y Byrne (2011), las redes de apoyo influyen positivamente en la calidad de vida y en el sentimiento de bienestar en las personas, aunque presenten diversas afecciones. Esto puede confirmarse en las investigaciones realizadas por Ferreira y Sherman (2007) quienes manifestaron que el apoyo social funciona como factor mediador entre el dolor y la presencia de síntomas depresivos. De igual forma, Kerns, Rosenberg y Otis (2002) coinciden con los demás autores expuestos en que el apoyo social es efectivamente un mediador en la percepción del dolor y la discapacidad, dado que, en su estudio, también identificaron que aquellos adultos que presentaban altos niveles de apoyo social, referían sentir menos dolor y lo afrontaban de mejor manera.
Otros estudios dentro de esta misma línea de la influencia del apoyo social en la percepción de la calidad de vida y la experiencia del dolor, refieren lo anteriormente encontrado. Por ejemplo, en un estudio sobre el estado de salud percibido, la satisfacción vital y el efecto moderador del apoyo social de 406 adultos mayores españoles se comprobó, que el apoyo social tuvo un efecto moderador en el impacto que la salud percibida tiene sobre la satisfacción vital de los participantes. En particular, aquellos participantes que indicaron tener una condición de salud muy mala pero que indicaron tener apoyo social (Dumitrache et al., 2016).

Por otra parte, en un estudio longitudinal en el cual se investigó la relación entre el estrés psicológico en pacientes recientemente diagnosticados con artritis reumatoide se encontró que cuanto más alto es el apoyo social y mientras menor es el dolor, menor sería el estrés psicológico de los pacientes (Benka et al., 2012). Lo anterior guarda relación con el estudio realizado por Ma et al. (2015) en el cual se demostraron correlaciones positivas entre el apoyo social percibido y la variable calidad de vida en adultos mayores con osteoporosis.

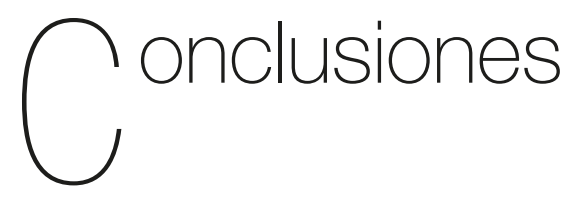

A partir de la investigación realizada en la cual se pretendía identificar las diferentes características del dolor osteomuscular y la percepción del apoyo social percibido en una muestra de adultos mayores, se logró identificar que las 
características del dolor varían entre los sujetos por diversos factores biológicos, psicológicos, culturales y sociales.

Respecto los datos sociodemográficos en cuanto a la variable sexo, se presentó un mayor número de mujeres que hombres, lo cual puede indicar que este género, desde esta investigación, reporta más dolor. Así mismo, se halló que la muestra señalaba niveles de ocupación relacionados con las tareas propias del hogar, lo cual guarda relación con el rol cultural con el que se le ha asociado al género. Por otro lado, se pudo evidenciar que el $37.5 \%$ de la muestra no terminó la básica primaria, lo que puede representar un factor de riesgo, ya que a menor nivel educativo mayor desconocimiento y menor uso de estrategias de afrontamiento y enfrentamiento para reconocer, atender, identificar y buscar soluciones frente a las dolencias presentadas. No obstante, se sugiere realizar investigaciones de corte correlacional para identificar si existen relaciones significativas entre el desconocimiento de las dolencias en el adulto mayor con el nivel educativo bajo.

Por último, en relación al apoyo social, se puede identificar que la muestra presenta altos niveles de apoyo en sus dimensiones: informacional, emocional, instrumental y relacional. Estos resultados permiten señalar que el apoyo social puede funcionar como mediador del padecimiento del dolor. Sin embargo, se sugiere realizar estudios correlacionales frente a estas variables para confirmar metodológicamente lo que teóricamente diversos autores refieren sobre la influencia del apoyo social en la percepción, manejo y evaluación del dolor.

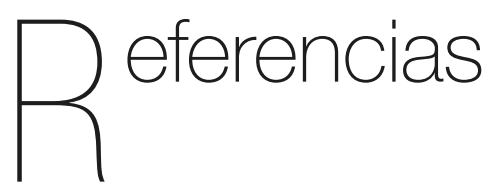

Alpízar, I. (2011). Jubilación y calidad de vida en la edad adulta mayor. Revista de la Facultad de Ciencias Sociales, 31(42), 15-26.

Álvarez-Namegyei, J., Nuño-Gutiérrez, BL., \& Alcocer-Sánchez, JA. (2005). Enfermedades reumáticas y discapacidad laboral en población adulta rural. Revista Médica del Instituto Mexicano del Seguro Social, 43(4), 287-292.

Amaya-Ropero, M., \& Carrillo-González, G. (2015). Apoyo social percibido y afrontamiento en personas con dolor crónico no maligno. Aquichan, 15(4), 461-474.

American Geriatrics Society (2002). The management of persistent pain in older persons. Journal of the American Geriatrics Society, 50(6), 205-224.

Asociación Colombiana para el Estudio del Dolor (2014). VIII Estudio Nacional de Dolor. Prevalencia del dolor crónico en Colombia. Recuperado de http://dolor.org.co/encuesta/ VIII\%20Estudio\%20Prevalencia\%20 dol or $\% 20$ cronic o $\% 20$ en $\% 20$ colombia\%20publicaci\%C3\%B3n\%20 p\%C3\%A1gina\%20ACED\%202014.pdf.

Bader, P., Echtle, D., Fonteyne, V., Livadas, K., De Meerleer, G., Paez, A., Papaioannou, E., \& Vranken, J. (2010). Guía clínica sobre el tratamiento del dolor. Recuperado de http://uroweb.org/wp-content/uploads/16GUIA-CLINICA-TRATAMIENTO-DOLOR1. pdf 
Benka, J., Nagyova, I., Rosenberg, J., Calffova, A., Mecejova, Z., Middel, B., Lazurova, I., P van Dijk, J., \& Gooothoff, J.W. (2012). Social support and psychological distress in rheumatoid arthritis: a 4 - year prospective study. Disability and Rehabilitation, 34(9), 754-761.

Bejarano, P., Osorio, R., Rodríguez, M., \& Berrío, G.(1985). Evaluación del dolor: adaptación del cuestionario de McGill. Revista Colombiana de Anestesiología, 13(4), 321-51.

Bistre, S. (2007). El dolor crónico en América Latina. Revista Iberoamericana del Dolor, 2(4), 7-9.

Brennan, F., Carr, D., \& Cousins, M. (2007). Pain Management: A Fundamental Human Right. Pain Medicine, 105(1), 205-221.

Català, E., Reig, E., Artés, M., Aliaga, L., López, JS., \& Segú, JL. (2002). Prevalence of pain in the spanish population: telephone survey in 5000 homes. European Journal of Pain, 6(2),133-40.

Clark, M., \& Cox, T. (2002). Refractory Chronic Pain. Psychiatric Clinics North America, 25(1),71-88

Comisión Económica para América Latina y el Caribe (CEPAL). (2006). Manual sobre indicadores de calidad de vida en la vejez. Recuperado de http://www.cepal.org/es/ publicaciones/3539-manual-indicadorescalidad-vida-la-vejez

Corujo, E., \& Pérez, D. (2007). Cambios más relevantes y peculiaridades de las enfermedades en el anciano. En varios autores (Eds.), Tratado de Geriatría para residentes. Sociedad Española de
Geriatría y Gerontología. Madrid, España: International Marketing \& Communication S.A.

Covarrubias-Gómez, A., Guevara-López, U., Lara-Solares, A., Tamayo-Valenzuela, AC., Salinas-Cruz, J., \& Torres-González, R. (2008). Características de los enfermos que acuden a clínicas del dolor por primera vez. Revista Médica del Instituto Mexicano del Seguro Social, 46(5), 467-472.

Cowan, D., Fitzpatrick, J., \& Roberts, J. (2003). The assessment and management of pain among older people in care homes: current status and future directions. International Journal of Nursing Studies, 40(3),291-298.

Curcio-Borrero, C. (2008). Soporte social informal, salud y funcionalidad en el anciano. Revista Hacia la Promoción de la Salud, 13, 42-58.

De Andrés, J., Acuña, J., \& Olivares, A. (2014). Dolor en el paciente de la tercera edad. Revista Médica Clínica Las Condes, 25(4), 674-686.

Díaz, R., Marulanda, F., \& Sáenz, X. (2009). Estudio epidemiológico del dolor crónico en Caldas, Colombia (Estudio Dolca). Acta Médica Colombiana, 34(3), 96-102.

Dumitrache, C.G., Rubio, L. \& Rubio- Herrera, R. (2016). Perceived health status and life satisfaction in old age, and the moderating role of social support. Aging and Mental Health, 20, 1-7.

Ferreira, V.M. \& Sherman, A.M. (2007). The relationship of optimism, pain and social support to well - being in older adults with osteoarthritis. Aging and Mental Health, 11(1), 89-98. 
Franks, H.M., Cronan, T.A. \& Oliver, K. (2004). Social support in women with fibromyalgia: ¿is quality more important than quantity? Journal of Community Psychology, 32(4), 425-438.

Gallardo-Peralta, L.P., Sánchez-Moreno, E., Arias-Astray, A. \& López-de Roda, A. B. (2015). Elementos estructurales de la red social, fuentes de apoyo funcional, reciprocidad apoyo comunitario y depresión en personas mayores en Chile. Anales de Psicología, 31(3), 1018-1029.

Gallegos-Carrillo, K., Mudgal, J., SánchezGarcía, S., Wagner, F.A., Gallo, J.J., Salmerón, J., \& García-Peña, C. (2009). Social networks and health-related quality of life: a population based study among older adults. Salud Pública México, 51(1),6-13.

Gibson, S. (2007). IASP global year against pain in older persons: highlighting the current status and future perspectives in geriatric pain. Expert Review of Neurotherapeutics, 7(6), 627-635.

Gloth, M. (2000). Geriatric pain. Factors that limit pain relief and increase complications. Geriatrics, 55(10), 46-54.

Guzmán, J., Huenchuan, S., \& Montes de Oca, V. (2003). Redes de apoyo social de las personas mayores: marco conceptual. 51 Congreso Internacional de Americanistas, Santiago de Chile, Julio de 2003.

Helme, R., \& Gibson, S. (2001). The epidemiology of pain in elderly people. Clinics in Geriatric Medicine, 17(3),417-431.

Herr, K., \& Garand, L. (2001). Assessment and measurement of pain in older adults. Clinics in geriatric medicine, 17(3), 457-478.
International Association for the Study of Pain. (1994). Subcommittee of Taxonomy of the International Association for the Study of Pain. In Merskey, H., \& Bogduk, N. (Eds.), Classification of Chronic Pain. (2da ed.). Seattle, USA: IASP Press.

Jaswal, N., \& Singh, S. (2014). Perceived social support among institutionalized and non - institutionalized elderly in Chandigarh (India). Indian Journal of Gerontology, 28(3), 372-374.

Kerns, R., Rosenberg, R. \& Otis, J. (2002). Self-appraised problem solving and painrelevant social support as predictors of the experience of chronic pain. Annals of Behavioral Medicine, 24(2), 100-105.

Krueger, A., \& Stone, A. (2008). Assessment of pain: A community-based diary survey in the USA. Lancet, 371, 1519-1525.

Ley N¹090. Por la cual se reglamenta el ejercicio de la profesión de Psicología, se dicta el Código Deontológico y Bioético y otras disposiciones. Bogotá, Colombia, 6 de septiembre del 2006.

Londoño, N., Rogers, H., Castilla, J., Posada, S., Ochoa, N., Jaramillo, M., Oliveros, M., \& Palacio., \& Aguirre-Acevedo, D. (2012). Validación en Colombia del cuestionario MOS de apoyo social. International Journal of Psychological Research, 5(1), 142-150.

López- Mongil, R., \& López, J. (2012). Prevalencia y fisiopatología del dolor crónico en el anciano. En Fernández et al. (Eds.), Guía de buena práctica clínica en geriatría. Dolor crónico en el anciano. Madrid, España: Sociedad Española de Geriatría y Gerontología. 
Ma, L., Li, Y., Wang J, Z.H., Yang, W., Cao, R., Qian, Y., \& Feng, M. (2015). Quality of life is related to social support in elderly osteoporosis patients in a Chinese Population. Plos One, 10(6), e0127849.

Maestre, J. (2001). El dolor en el adulto mayor. Colombia Médica, 32(4), 184-188.

Martínez- Salio, A. (2007). Anamnesis del paciente con dolor neuropático. En Serra, C. (Ed.), Tratado de dolor neuropático. Madrid, España: Médica Panamericana.

Matud, P., Carballeira, M., López, M., Marrero, R., \& Ibáñez, I. (2002). Apoyo social y salud: un análisis de género. Salud Mental, 25(2), 32-37.

Maxwell, C., Dalby, D., Slater, M., Patten, S., Hogan, D., Eliasziw, M., \& Hirdes, J. (2008). The prevalence and management of current daily pain among older home care clients. Pain, 138(1), 208-16.

McWilliams, L., Brian J., \& Murray, W. (2003). Mood and anxiety disorders associated with chronic pain: an examination in a nationally representative simple. Pain, 106(1), 127-33.

Noceda, J., Moret, C., \& Lauzirika, I. (2006a). Características del dolor osteomuscular crónico en pacientes de atención primaria. Resultados de un centro rural y otro urbano. Revista de la Sociedad Española del Dolor, 13(5), 287-293.

Noceda, J., Moret, C., \& Lauzirika, I. (2006b). Calidad de vida en el dolor osteomuscular crónico. Reumatología Clínica, 2(6), 298-301.

Ordóñez-Hernández, C., ContrerasEstrada, M., \& Soltero-Avelar, R. (2015).
Mujeres trabajadoras con dolor crónico osteomuscular: análisis de caso. Cadernos de Saúde Pública, 31(10), 2223-2233.

Peláez, B., \& Rodríguez-Wong, L. (2004). Vejez y recursos sociales en América Latina. Humanitas: Humanidades Médicas, (1), 73-83.

Pelcastre-Villafuerte, B., Treviño-Siller, S. González-Vázquez, T., \& Márquez-Serrano, M. (2011). Apoyo social y condiciones de vida de adultos mayores que viven en la pobreza urbana en México. Cadernos de Saúde Pública, 27(3), 460-470.

Plata, L., \& Cerquera, A. (2016). Dolor osteomuscular y apoyo social en una muestra de gerontes de Bucaramanga. Pensamiento Psicológico, 14(2), 125-135.

Robinson, C. (2007). Relieving pain in the elderly. Health Prog, 88(1), 48-53.

Rodrigo, M.J. \& Byrne, S. (2011). Social support and Personal agency in At-Risk Mothers. Psychosocial Intervention, 20(1), 13-24.

Sánchez, B. (2003). Abordajes teóricos para comprender el dolor humano. Revista Aquichan, (3), 32-41.

Soto, H. (2011). Dolor: aspectos generales, conceptualización e intervención. (Tesis de pregrado). Universidad de la Sabana, Bogotá.

Terman, G., \& Bonica, J. (2001). Spinal Mechanisms and their modulation. En Bonica's management of pain., \& Loeser, JD. (Eds.), Bonica's textbook of pain management. (3ra ed.). Philadelphia, USA: LWW. 
Torvik, K., Kaasa, S., Kirkevold, O., \& Rustoen, T. (2009). Pain and quality of life among residents of Norwegian nursing homes. Pain Management Nursing, 11, 35-44.

Trujillo, C. (2008). Epidemiología del dolor en el adulto mayor. En Fernández, C. (Ed.), Dolor en el adulto mayor. Bogotá, Colombia: Asociación Colombiana para el Estudio del Dolor, ACED.
Villarreal, G., \& Month, E. (2012). Condición sociofamiliar, asistencial y de funcionalidad del adulto mayor de 65 años en dos comunas de Sincelejo (Colombia). Revista Científica Salud Uninorte, 28(1), 75-87.

Vivaldi, F. \& Barra, E. (2012). Bienestar psicológico, apoyo social percibido y percepción de salud en adultos mayores. Terapia Psicológica, 30(2), 23-29. 